\title{
Investigations on Multi Pumped Fiber Raman Amplifiers over WDM in Optical Communication System
}

\author{
Kulwinder Singh \\ UCoE, Punjabi University, \\ Patiala, Punjab, India.
}

\author{
Manjeet Singh Patterh \\ UCoE, Punjabi University, \\ Patiala, Punjab, India.
}

\author{
Manjit Singh Bhamrah \\ UCoE, Punjabi University, \\ Patiala, Punjab, India.
}

\begin{abstract}
Fiber Raman amplifiers are important component of wavelength division multiplexed fiber-optic communication systems. The number of pumps, determination of powers, wavelengths and pumping schemes are main design criteria to operate these amplifiers. This paper investigates the effect of counter propagating pumping in fiber Raman amplifier. Pumping options like single, two and seven counter propagating pumps are investigated and their effect on bandwidth and gain ripple and signal power conditions are explored. It is shown that optical bandwidth increases from $5.9 \mathrm{THz}(45.3 \mathrm{~nm})$ in the range $1535-1584 \mathrm{~nm}$ to $12.0 \mathrm{THz}$ $(97.6 \mathrm{~nm})$ in the range $1511-1607 \mathrm{~nm}$ and gain ripple decreases to 0.47 from 0.82 by increasing the number from one to seven counter propagating pumps in the fiber Raman amplifier.
\end{abstract}

\section{General Terms}

Fiber Raman amplifier, counter pumping, gain-bandwidth, Raman gain flatness,

\section{Keywords}

Multi- pumping fiber Raman amplifier, optimization of gain flatness.

\section{INTRODUCTION}

Modern lightwave communication systems have used full gain bandwidth of erbium doped fiber amplifiers (EDFAs), and increasing capacity has resulted into the need of broader bandwidth optical amplifiers in the systems [1]. Fiber Raman amplifiers (FRAs) are used nowadays as all Raman or hybrid FRAs/EDFAs in all long haul and ultra long haul wavelength division multiplexed (WDM) optical communication systems [3]. FRAs have certain advantages such as wavelength flexibility, because gain depends on the frequency shift between pump and signal, multi pumped fiber Raman amplifiers have more bandwidth than erbium doped fiber amplifiers (EDFAs). Moreover, Raman amplifiers offer better noise performance than EDFAs because they are inherently distributed amplifiers. The design process of fiber Raman amplifiers involves the criteria of number of pumps, pumping schemes, selection of their powers, frequencies (wavelengths) and cost and complexity etc. The bandwidth of distributed multi pump Raman amplifier can be increased by increasing the number of pumps because of total composite gain is due to individual pump waves at different wavelengths [3]. In other way, WDM pumping can also be used to generate a flat gain [4]. The Gain flattening with broad gain bandwidth at the operating wavelength range of multi pumped fiber Raman amplifier is a challenge to the researchers $[1,2]$. So far research works reported deal with analysis and solution of Raman amplifier modeled equations [5]. While G. D. Podder et. al. has designed Raman amplifier with four co-propagating pumps for $65 \mathrm{~nm}$ gain bandwidth and gain ripple of 0.9 using simulated models [8]. M. N. Islam et. al. has proposed a design of multi-pumped FRAs using co, counter, bidirectional propagating taking two pumps and for a distributed pumping scheme [6]. The problem is still open for the optimization and gain flattening of multi pumped fiber Raman at varied pump powers and wavelengths. So, the paper is further extension of $[6,8]$, by proposing a design of multi pumped fiber Raman amplifier taking varied pump powers and its wavelengths using counter propagating scheme with one to seven pumps. The novelty of the work is that it achieved a uniform gain spectrum of the amplifier for a broad gain bandwidth.

The paper in the section 1, presents introduction of multi pumped fiber amplifiers, section 2 discusses theoretical model prevalent in the area. The section 3 describes schematic of multi pumping distributed amplifier while section 4 gives results \& their discussion. Finally in section 5, conclusions of the work done have been drawn.

\section{THEORETICAL MODEL}

Raman amplifier is based on the principle of stimulated Raman scattering (SRS) effect in an optical fiber where a strong pump wave at shorter wavelength provides gain to signal waves at longer wavelengths. The difference in energy of scattered photons appears as optical phonons (molecular vibrations). Raman gain spectrum in fused silica fibers spreads over $40 \mathrm{THz}$ bandwidth as shown in figure 1 [7]. The gain spectrum shifts with the pump spectrum, while the peak gain coefficient around $13.2 \mathrm{THz}$ varies inversely with the pump wavelength [6]. The telecommunications bands are around $1500 \mathrm{~nm}$, so $13.2 \mathrm{THz}$ frequency shift amounts to approximately $100 \mathrm{~nm}$ wavelength shift. Mathematically, the interaction between the pump and signal propagating in fiber is governed by the following two coupled equations [8].

$$
\begin{aligned}
& \frac{d S\left(f_{s}, z\right)}{d z}=\frac{g_{r}\left(f_{s}, f_{p}\right) S\left(f_{s}, z\right) P\left(f_{p}, z\right)}{k A_{\text {eff }}}-\alpha_{s} S\left(f_{s}, z\right) \\
& \frac{d P\left(f_{p}, z\right)}{d z}=-\frac{f_{p}}{f_{s}} \frac{g_{r}\left(f_{s}, f_{p}\right) S\left(f_{s}, z\right) P\left(f_{p}, z\right)}{k A_{e f f}}-\alpha_{p} P\left(f_{p}, z\right)
\end{aligned}
$$




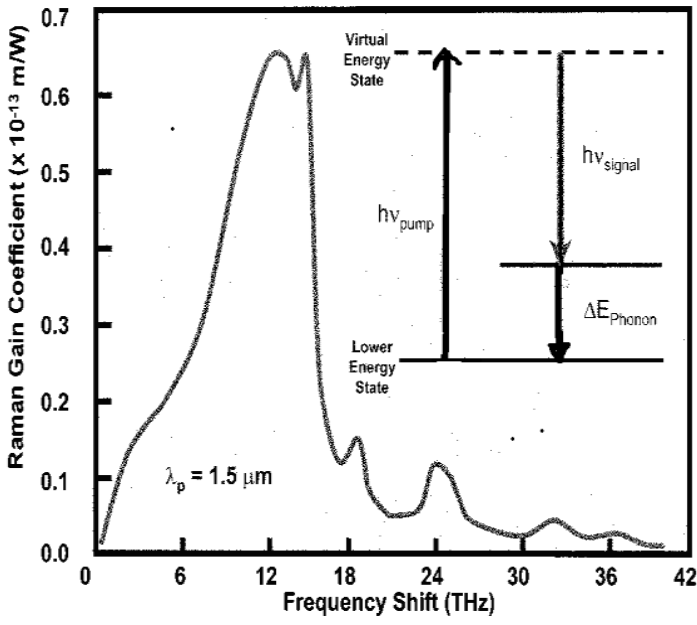

Figure 1 Raman gain curve for a silica-core single mode fiber.

where $S\left(f_{s}, z\right)$ is the signal power at frequency $f_{s} ; P\left(f_{p}, z\right)$ is the pump power at frequency $f_{p} ; \alpha_{\mathrm{s}}$ and $\alpha_{\mathrm{p}}$ are the attenuation constants for the signal and pump respectively; $g_{r}\left(f_{p}, f_{s}\right)$ is the Raman gain coefficient which depends on the frequency shift between the pump and signal, $\Delta f=f_{p}-f_{s}$. $A_{e f f}$ is the effective cross sectional area of the fiber and $k$ is the polarization constant, which is 1 if the signal and pump are polarized and 2 if not polarized. The first term in Equation (2) represents the pump depletion due to the SRS effect, which can be neglected. Solving Equations (1) and (2), the Raman gain of a signal can be determined. as

$$
\begin{aligned}
& G_{r}=\exp \left[\frac{g_{r}\left(f_{s}, f_{p}\right) P\left(f_{p}, z\right) L_{\text {eff }}}{k A_{\text {eff }}}\right] \\
& G_{r}(\text { ind } d B)=4.43 \frac{g_{r}\left(f_{s}, f_{p}\right) P\left(f_{p}, z\right) L_{e f f}}{k A_{e f f}}
\end{aligned}
$$

Where $L_{\text {eff }}=\left\{1-\exp \left(-\alpha_{p} L\right)\right\} / L$ represents the effective fiber length taking into account the fiber loss at pump wavelength.

On the other hand, a number of other factors may be considered for detailed designing a Raman amplifier with multi-wavelength pumps for DWDM systems like pump-to pump power transfer, signal-to-signal power transfer, pump depletion, Rayleigh back-scattering, amplified spontaneous emission (ASE) noise, loss due to noise emission, FWM in signals and pumps. The some of above factors may not be required depending on the contribution towards a specific problem.

\section{SYSTEM DESCRIPTION}

A simulation model of multi pumped fiber Raman amplifier with counter propagating pumps is designed as shown in Figure 2. To simplify the analysis, signal-signal, pump-pump power transfer, pump depletion, loss due to noise transfer and four wave mixing (FWM) in signals and pumps in this model have not been considered as they have very less effect on the gain spectrum equalization [2]. To analyze the model, eight WDM channels at $10 \mathrm{~Gb} / \mathrm{s}$ and with $100 \mathrm{GHz}$ spacing, are launched simultaneously in three different branches. In each branch of them, a $25 \mathrm{~km}$ fiber link with loss $=0.2 \mathrm{~dB} / \mathrm{km}$ and dispersion parameter $\mathrm{D}=-2 \mathrm{ps} / \mathrm{nm} / \mathrm{km}$ is used. The channel frequencies $(\mathrm{THz})$ and wavelengths $(\mathrm{nm})$ of the model considered are shown in the table 1 . The table 2 shows the wavelength $(\mathrm{nm})$ and pump powers $(\mathrm{mW})$ for single, two and seven pumping schemes. The selected wavelengths and powers of pumps are the optimized for obtaining the broadened the gain-bandwidth and flat gain. The upper branch in the schematic, the fiber is pumped by a $500 \mathrm{~mW}$ single counter-propagating pump at $1465 \mathrm{~nm}$. Secondly, middle fiber, it is pumped by two counter-propagating pumps ( $\mathrm{P}=$ 200 mW @ 1427 nm and P = 300 mW @ 1495 nm). Lastly, lower branch, the fiber is pumped by seven pumps in the range from 1427-1495 $\mathrm{nm}$. These pump wavelengths are useful for the amplification of $\mathrm{C}$ and $\mathrm{L}$ bands of optical transmission systems.

\section{RESULTS AND DISCUSSION}

The optical communication system model illustrated in figure 2 is investigated with backward pumping employing single, two and seven counter propagating pump laser diodes to achieve a gain in wavelength range 1535-1630 nm with minimum possible gain ripple. The investigations of on-off gain $(\mathrm{dB})$ versus optical signal frequencies $(\mathrm{THz})$ are as shown in figure 3 , where dotted curve is showing the gain for single counter propagating pump, dot-dash curve of two counter propagating pumps and solid curve is drawn for the gain of Raman amplifier with seven counter propagating pumps. By comparing these results, it is evident that there is a change in the Raman gain with increase of number of pumps. In the seven pumps case a quite flat gain is obtained in the bandwidth occupied by the 8 channels. The results are computed quantitatively and shown table 3 in addition to figure 3. It indicates that Raman amplifier gain bandwidth and gain flatness or gain ripple for single, two and seven counter propagating pumps. The results provides that gain bandwidth is $5.965 \mathrm{THz}$ for single, $7.210 \mathrm{THz}$ for two and $12.102 \mathrm{THz}$ for seven pumps and gain flatness is 0.82 for single, 0.56 for two and 0.47 for seven pumps. So by increasing the number of counter propagating pumps the gain bandwidth increases regularly and it makes amplifiers more flat. The measured optical spectral density of the signals at the transmitter and receiver ends using optical spectrum analyzer in figure 4 . The Figure 4 (a) shows the optical spectral density at transmitter of the fiber Raman amplifier while figure 4 (b), 4(c) and 4(d) indicate the output for single, two and seven counter propagating pumps at the receiver respectively. On comparison, the optical spectral densities for different configurations, clearly gives snapshot of optical signal moderately equalized by increasing the number of pumps. Their performance comparison have summarized for the configurations in table 4 , where optical spectrum peak powers of 8 channels at input and output are shown for single, two and seven counter propagating pumps. Collectively, looking at the figure $3,4(\mathrm{~b}), 4(\mathrm{c})$ and $4(\mathrm{~d})$ as well as entries shown in tables 3 and 4, it may summed up that optical bandwidth increases from $5.965 \mathrm{THz}(195.485-185.519 \mathrm{THz})$ to 12.102 $\mathrm{THz}(198.832-186.730 \mathrm{THz})$ and gain ripple decrease from 0.82 to 0.47 by increasing the number of pumps from one to seven counter propagating in the same fiber of Raman amplifier. The behaviour of signal and pump wave along the Raman fiber length have been plotted. These evolutions are shown in figure 5 (a) for single pump, 5 (b) for two pumps and 5 (c) for seven pumps. The comparison these evolution curves endorses the findings that the seven pumps give increased uniform amplification and broader gain bandwidth. 
Table 1 Eight WDM signal channel frequencies and wavelengths.

\begin{tabular}{|c|c|c|c|c|c|c|c|c|}
\hline Channel No. & 1 & 2 & 3 & 4 & 5 & 6 & 7 & 8 \\
\hline Signal frequency (THz) & 190.65 & 190.75 & 190.85 & 190.95 & 191.05 & 191.15 & 191.25 & 191.35 \\
\hline Signal wavelength (nm) & 1572.47 & 1571.65 & 1570.82 & 1570.00 & 1569.18 & 1568.36 & 1567.54 & 1566.72 \\
\hline
\end{tabular}

Table 2 Pump Wavelengths and pump powers for one, two and seven pumps

\begin{tabular}{|c|c|c|c|c|c|c|c|c|c|}
\hline \multicolumn{2}{|c|}{ Pump Wavelength(nm) } & 1427 & 1439 & 1450 & 1464 & 1465 & 1478 & 1485 & 1495 \\
\hline \multirow{3}{*}{$\begin{array}{c}\text { Pump } \\
\text { Power } \\
(\mathrm{mW})\end{array}$} & Single pump & - & - & - & - & 500 & - & - & - \\
\cline { 2 - 11 } & Two pumps & 200 & - & - & - & - & - & - & 300 \\
\cline { 2 - 11 } & Seven pumps & 130 & 120 & 110 & 100 & - & 90 & 100 & 120 \\
\hline
\end{tabular}

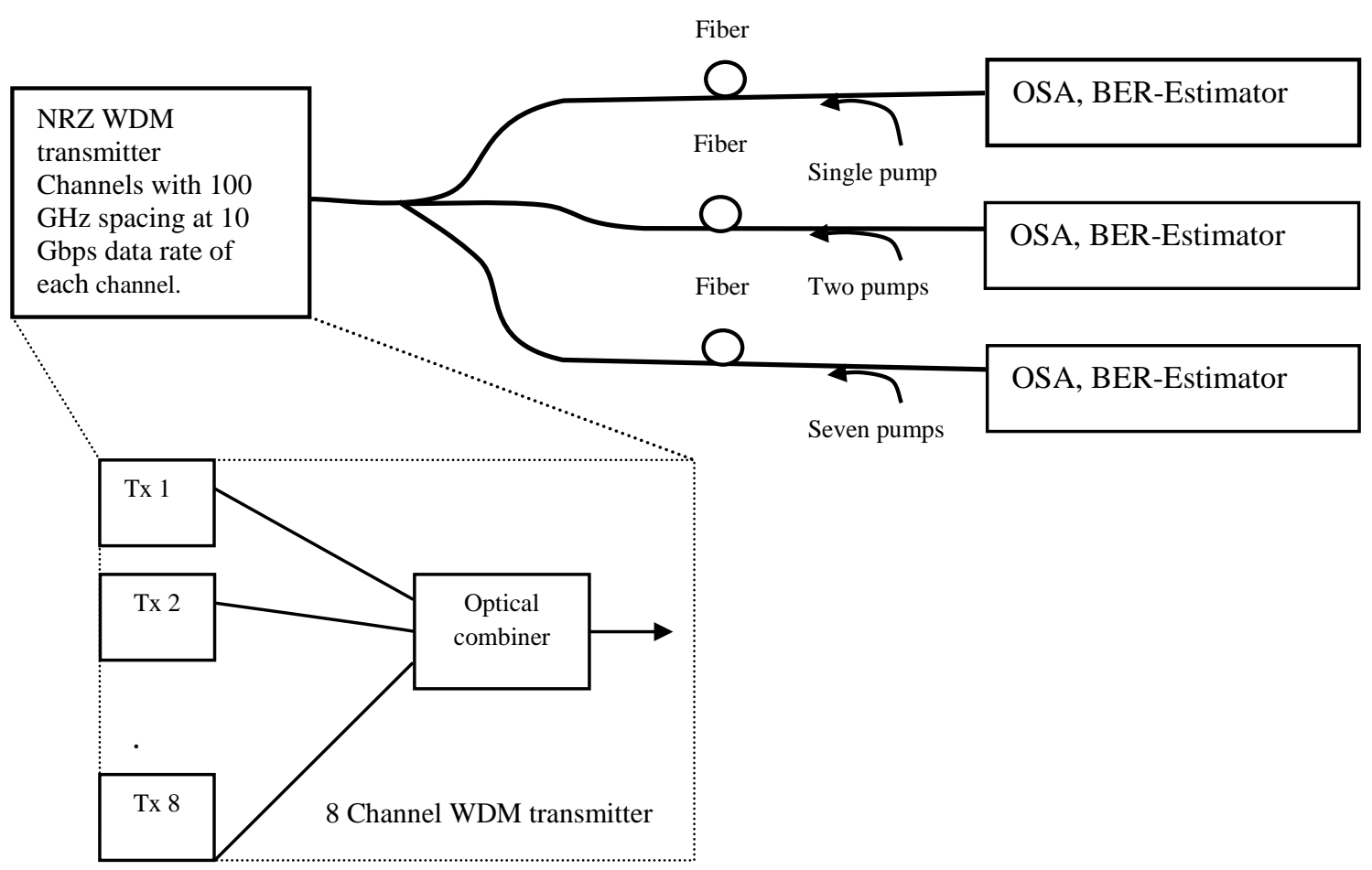

Figure 2 Optical communication system model of 8 channel WDM optical network at 10Gb/s with 100 GHz channel spacing employing Raman amplifier. 


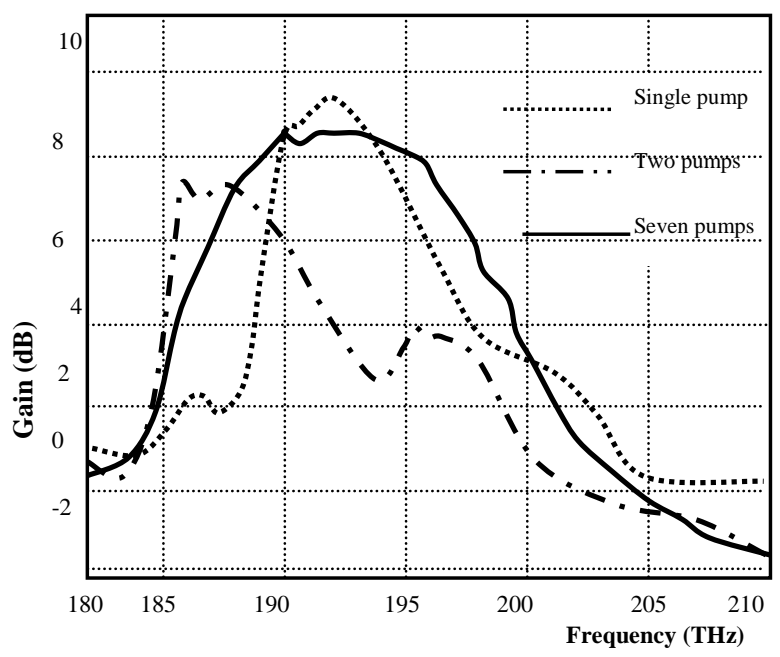

Figure 3 Gain (dB) versus frequency (THz) plot of Raman amplifier. Dotted curve for single, dot -dash curve for two and solid curve for seven pumps.
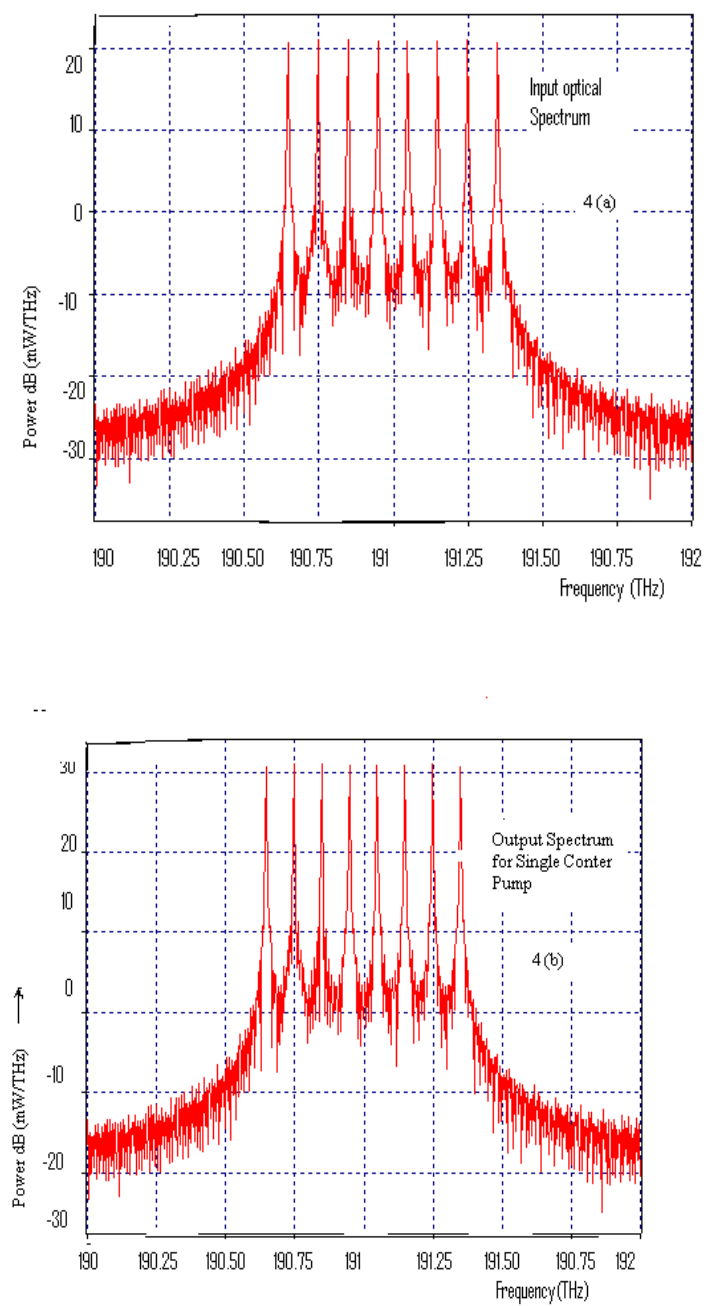

Table 3 Gain-bandwidth and gain ripple of FRA

\begin{tabular}{|c|c|c|c|c|}
\hline S. & $\begin{array}{c}\text { No. of } \\
\text { counter } \\
\text { pumps }\end{array}$ & $\begin{array}{c}\text { Gain } \\
(\mathrm{dB})\end{array}$ & $\begin{array}{c}\text { Gain } \\
\text { Bandwidth } \\
(\mathrm{THz})\end{array}$ & $\begin{array}{c}\text { Gain } \\
\text { Flatness or } \\
\text { gain ripple }\end{array}$ \\
\hline 1 & $\begin{array}{c}\text { One } \\
\text { (dotted } \\
\text { Curve) }\end{array}$ & 9.2 & $\begin{array}{c}195.485 \text { to } \\
185.519 \\
(\Delta \mathrm{f}=5.965)\end{array}$ & 0.82 \\
\hline 2 & $\begin{array}{c}\text { Two } \\
\text { (dot-dash } \\
\text { curve) }\end{array}$ & 7.2 & $\begin{array}{c}192.438 \text { to } \\
185.185 \\
(\Delta \mathrm{f}=7.210)\end{array}$ & 0.56 \\
\hline 3 & $\begin{array}{c}\text { Seven } \\
\text { Pumps } \\
\text { (Solid } \\
\text { curve) }\end{array}$ & 8.2 & $\begin{array}{c}198.832 \text { to } \\
186.730 \\
(\Delta \mathrm{f}= \\
12.102 \mathrm{~nm})\end{array}$ & 0.47 \\
\hline
\end{tabular}
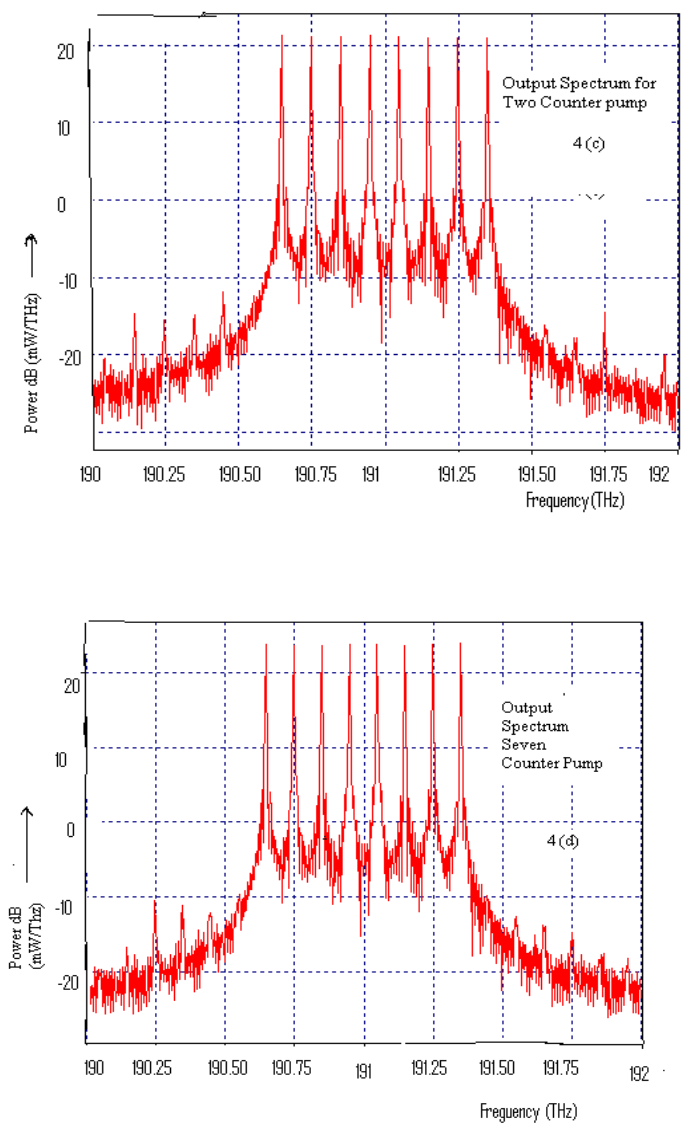

Figure 4. Power Spectrums at the input of SMF $25 \mathrm{~km}$ figure (a). Output power spectrum for counter propagating pumps number of (b) one, (c) two and (d) seven. 


\section{CONCLUSION}

The multi pumped fiber Raman amplifier have been investigated to optimize its performance using various pump wavelengths and powers under counter propagating pumping configurations. In general, it is seen that the optical gain bandwidth of Raman amplifier increases by increasing the number of counter propagating pumps which results a decrease in gain ripple in optical gain spectrum of these amplifiers. Specifically, with seven counter propagating pumps gain-bandwidth increases to $12.102 \mathrm{THz}$ and gain ripple decreases to 0.47 . This achievement in characteristics for wide band fiber Raman amplifiers could be utilized in applications of modern WDM optical networks and systems. if little noise generated can be compromised.
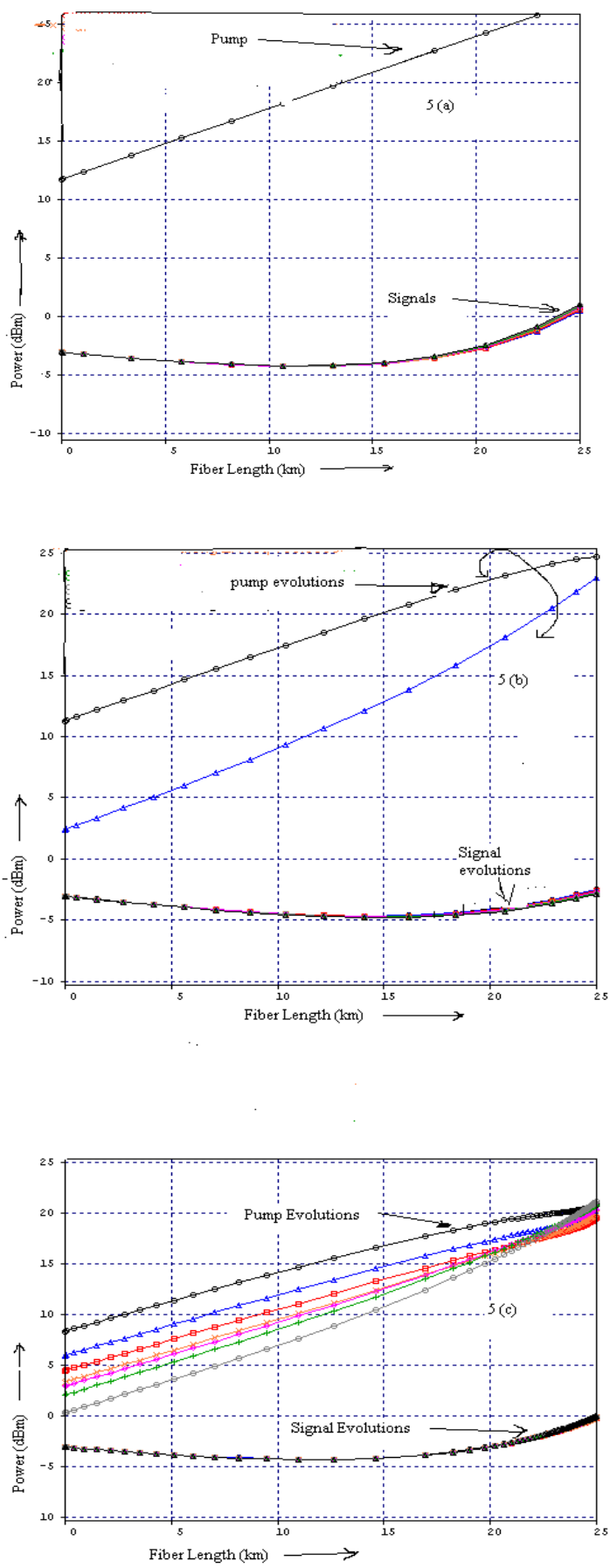

Figure 5 :Pump and signal evolutions for 5 (a) Single pump 5 (b) Two pumps 5(c) Seven pumps
Table 4 Optical Spectrum at input and output of 8 hannels.

\begin{tabular}{|c|c|c|c|c|c|}
\hline \multirow{2}{*}{$\begin{array}{l}\text { S. } \\
\text { No }\end{array}$} & \multirow{2}{*}{$\begin{array}{c}\text { Frequen } \\
\text { cy } \\
(\mathrm{THz})\end{array}$} & \multirow{2}{*}{$\begin{array}{c}\text { Input } \\
\text { Power } \\
\text { dB } \\
(\mathrm{mW} / \\
\mathrm{THz})\end{array}$} & \multicolumn{3}{|c|}{$\begin{array}{c}\text { Output } \\
\text { Power dB (mW/THz) }\end{array}$} \\
\hline & & & $\begin{array}{l}\text { Single } \\
\text { pump }\end{array}$ & $\begin{array}{l}\text { Two } \\
\text { Pump }\end{array}$ & $\begin{array}{l}\text { Seven } \\
\text { pump }\end{array}$ \\
\hline 1 & 190.652 & 20.7291 & 23.956 & 21.4393 & 24.2557 \\
\hline 2 & 190.749 & 21.0109 & 23.5966 & 21.3104 & 24.356 \\
\hline 3 & 190.848 & 21.0109 & 23.5916 & 21.3104 & 24.6166 \\
\hline 4 & 190.952 & 21.7291 & 23.8316 & 21.4393 & 24.7469 \\
\hline 5 & 191.049 & 21.0109 & 23.8316 & 21.1816 & 24.7469 \\
\hline 6 & 191.15 & 21.0109 & 23.8316 & 20.9239 & 24.7469 \\
\hline 7 & 191.25 & 21.0109 & 24.8316 & 20.9239 & 24.7469 \\
\hline 8 & 191.349 & 20.87 & 24.8316 & 21.0527 & 24.8772 \\
\hline
\end{tabular}

\section{REFERENCES}

[1] Jonathan Hu, B. S. Marks and C. R. Menyuk "Flat gain Fiber Raman amplifiers using equally spaced pumps" J. of Lightwave Technology, vol. 22, no.6, pp. 15191522, June. 2004.

[2] V. Perlin and H. Winful, "Optimal design of flat-gain wide-band fiber Raman amplifiers," J. Lightwave Technol., vol. 20, no. 2, pp. 250-254, Feb. 2002.

[3] L X Ming, L Y He "Optimal bandwidth for distributed Multi pump Raman Amplifier based on hybrid Genetic Algorithm." China. Phys. Letters. vol. 21, no.1, 2004.

[4] M. Ketabi Jahromi and F. Emami "Simulation of Distributed Multi pump Raman Amplifier in Different Transmission media" International Journal of communications, Issue 4, vol.2, 2008.

[5] A.R Bahrampour, A. Ghasempour and L.Rashmi "Gain ripple minimization in fiber Ramann amplifiers basd on variation method" Elsevier, Optics Communications, 1545-1557, 2008.

[6] M. N. Islam and M.S. Alam "Design of Raman amplification with multiple pumping for optical DWDM systems." $3^{\text {rd }}$ ICECE 2004, 28-30 December, 2004, Dhaka, Banladaesh.

[7] M. N. Islam, "Raman amplifiers for telecommunications," IEEE J. Select. Topics Quantum Electron.”, vol. 8, no. 3, pp. 548-559, 2002.

[8] G D Podder and M N Islam "Uniform gain of a multi wavelength pumped wideband Raman amplifier optical communication System", $3^{\text {rd }}$ ICECE 2004, 28-30 December, Dhaka, Bangladesh.

[9] J. Bromage, "Raman amplification for fiber communications systems," IEEE Journal of Lightwave Technology., vol. 22, no. 1, pp. 79-93, 2004. 